\title{
El espíritu empresarial desde las representaciones sociales: caso Venezuela ${ }^{1}$
}

\author{
Managerial Spirit from de Social Representations: Venezuelan case
}

\author{
Leslie Mercedes Borjas De Xena \\ Universidad Metropolitana de Caracas, Venezuela \\ lborjas@unimet.edu.ve
}

Artículo de investigación científica y tecnológica recibido el 18/03/10 y aprobado el 31/05/10

\begin{abstract}
Resumen
El objetivo de este trabajo consiste en caracterizar las estructuras centrales y periféricas de las representaciones sociales (RS) sobre el espíritu empresarial (EE). Las RS son formas de pensamiento social que incluyen informaciones, creencias, actitudes, prácticas y experiencias. Para el análisis se usaron 65 artículos -en el período 2006 al 2009- y un buen número de entrevistas a profundidad. Los resultados evidenciaron el anclaje de las RS en el contexto venezolano, que lo caracteriza "altamente" emprendedor.
\end{abstract}

Palabras clave: Representaciones sociales, Espíritu empresarial, Cultura nacional, Medios impresos.

\begin{abstract}
This research seeks to characterize the central and peripheral structures of the Social Representations (SR) on Entrepreneurial Spirit (ES). The SR are ways of social thinking that include information, beliefs, attitudes, practices and experiences. This type of research is complementary. The methodology called for the analysis of content found in secondary literature and in depth interviews. The corpus includes 65 articles in the 2006-2009 period. The results showed that the anchorage of the SR in the Venezuelan context could be characterized as "highly" entrepreneur.
\end{abstract}

Key words: Social Representations, Managerial Spirit, National Culture, Press Media.

1 Este trabajo de investigación fue realizado como parte de la línea de investigación "Emprendedores y empresas familiares", de la Universidad Metropolitana de Caracas. 



\section{Introducción}

El espíritu empresarial es considerado, para efectos de este trabajo, una competencia a nivel personal que se desarrolla en organizaciones públicas y privadas, que es clave en la generación de organizaciones competitivas.

Kantiss, Angelelli y Moori (2004:10) sostienen que en los países de América Latina se "está produciendo una verdadera transformación en las políticas de desarrollo empresarial que incorpora el fomento de nuevos emprendimientos y la promoción de una nueva mentalidad que valoriza la actividad empresarial".

Borjas, Eugui, Marvez, Monasterio (2010) definen iniciativa empresarial como una idea innovadora, a través de la creación de una organización para explotar una oportunidad detectada en el ambiente. Esta definición hace énfasis en varios aspectos: la señala como una actividad y no como un atributo o característica; esta actividad puede ser personal o colectiva de un equipo de personas; es una actividad creativa cuyo proceso está comprendido por la detección de la oportunidad, la generación de la idea para dar respuesta a esa oportunidad y el diseño e implantación de una organización dirigida a explotar sistemáticamente esa oportunidad. La definición comprende términos suficientemente amplios que integra actividades cuyo fin o propósito fundamental no sea el mercantil, a través de organizaciones no gubernamentales.

Hayton y Kelley (2006), conciben al espíritu empresarial como una ventaja competitiva, que involucra una serie de actividades tales como: innovación y el desarrollo de corporativas internas y externas en productos y procesos.

El modelo de las representaciones sociales tiene un carácter interdisciplinario y funcional, por su utilidad en la interpretación de los fenómenos en la sociedad actual. Centra su interés en el estudio de los significados que distintos actores sociales tienen sobre una situación o evento presente en el entorno. Banchs (2005), en la investigaciones realizadas para la comprensión y aplicación de la teoría, las define como expresiones de valores, creencias y opiniones que son reguladas por las normas sociales de cada colectividad y son de gran utilidad para comprender los significados, los símbolos y las formas de interpretación que los seres humanos utilizan para referirse a objetos o eventos.

Las representaciones sociales (Jodelet, 2001 - 2004), en tanto proceso social, sólo pueden aparecer en grupos y sociedades en las que el discurso incluye la interacción de diferentes actores. El lenguaje de los diferentes actores y el usado por los medios son formas de transmisión, comunicación y permanente reconstrucción de símbolos y significados. Son esta experiencia y conocimiento contradictorios los que permiten el tipo de discurso colectivo que crea, en las sociedades modernas, lo que llamamos conocimiento ordinario y sentido común. 
En el proceso de conversación y en los medios de comunicación de masas, los objetos sociales son creados y elaborados por actores sociales que pueden tomar parte en el proceso de comunicación, mediante cualquiera de los medios que posean.

Las representaciones sociales son un modelo que tiene por función conceptualizar lo real a partir del conocimiento previo, designa una forma específica de conocimiento: "el saber de sentido común", en el que el contenido significa una forma particular de pensamiento social.. Por consiguiente, son tipos de creencias paradigmáticas, organizaciones de creencias, organizaciones de conocimiento y lenguajes que conforman distintas estructuras de conocimiento.

A Moscovici se le atribuye la conceptualización del constructo, el cual define como:

[...] un sistema de valores, ideas y prácticas que tienen una doble función: en primer lugar, establecer un orden que permita a los individuos orientarse en su mundo social y material y en segundo término, permitir la comunicación entre los miembros de una comunidad, aportándoles un código para el intercambio social y para denominar y clasificar de manera inequívoca los distintos aspectos de su mundo y de su historia individual y grupal (Moscovici, 1973: XIII ).

Para Vasilachis de Gialdino (2003: 268) las representaciones sociales son construcciones simbólicas individuales y/o colectivas que los sujetos ven o a las que los sujetos apelan para interpretar al mundo, reflexionar sobre su propia situación y la de los demás y determinar el alcance y la posibilidad de su acción histórica.

Los procesos que describen el funcionamiento y la generación de representaciones sociales son: la objetivación y el anclaje. La objetivación se refiere a la trasformación de conceptos abstractos, extraños en experiencias o materializaciones concretas. El anclaje permite incorporar lo extraño, en una red de categorías y significaciones, a través de dos modalidades de intervención: a) la inserción del objeto de representación en un marco de referencia conocido y preexistente; b) la instrumentalización social del objeto representado que posibilita la inserción de las representaciones en la dinámica social haciéndolas instrumentos útiles de comunicación y comprensión, que posibilitan que las personas puedan comunicarse en los grupos a que pertenecen bajo criterios comunes, con un mismo lenguaje para comprender los acontecimientos, las personas u otros grupos.

Abric (2001), sugiere que las representaciones sociales están organizadas alrededor de un núcleo y de unos elementos periféricos. El núcleo central (principio generador) tiene dos funciones esenciales: una función mediante la cual los otros 
elementos de la representación adquieren o trasforman su significado y una función organizadora de las relaciones que asocia los elementos de la representación. Este núcleo es la parte más estable, coherente y rígida de la representación, ya que está fuertemente anclado sobre la memoria colectiva del grupo que lo elabora, tiene una función consensual y define la homogeneidad compartida por el grupo, estableciendo un carácter normativo de las significaciones. Los elementos periféricos se encuentran coordinados como un conjunto que se opone al sistema nuclear o central; permiten la regulación y la adaptación de las características del sistema central a la situación concreta que un grupo enfrenta.

Los medios impresos, en este caso la prensa, reflejan el sentido histórico y tienen un peso preponderante para transmitir valores, conocimientos, creencias y modelos de conducta que tiene un evento de manera estructurada; expresan a través de sus discursos, dan cuenta de las principales formas de representación de un hecho en diferentes ámbitos de la sociedad, por lo que el estudio persigue caracterizar cómo el evento estudiado es visto a través de diferentes perspectivas los medios impresos, editores y expertos en educación empresarial. El discurso de los medios impresos se refiere a un objeto, a un mundo que se pretende describir, expresar y construir. Tiene la característica de social, porque las configuraciones ya elaboradas se transforman y se convierten en significados autónomos.

En Venezuela existen organizaciones educativas y empresariales que desarrollan las competencias del espíritu empresarial, entendido como una competencia básica para el apoyo de nuevas exigencias y transformaciones en los procesos y servicios de la colectividad. Dentro de este contexto, la investigación tiene relevancia puesto que permite caracterizar la representación social sobre el espíritu empresarial que está presente en los medios de comunicación, actores que comparten con las instituciones educativas, un rol en la producción, registro del capital cultural de un grupo social, en un contexto histórico, fundamentalmente porque en nuestro medio no se conocen experiencias previas que caractericen el significado del espíritu empresarial en contextos diferentes a los ya tradicionalmente considerados.

El estudio tiene como interés develar las representaciones sociales del espíritu empresarial latinoamericano que emerge del imaginario de los actores que se desenvuelven en el medio venezolano, las cuales se elaboran y naturalizan, a través de la objetivación y el anclaje. En este orden de ideas, al identificar los elementos constitutivos de la representación social sobre espíritu empresarial se logra conocer la organización de estos elementos e identificar el núcleo central de la representación. De acuerdo a estas consideraciones las preguntas que guían la investigación son: 
¿Cuáles representaciones sociales del espiritu empresaria prevalecen en el discurso de la prensa nacional?: Cuáles son, los temas más difundidos relativos a las representaciones sociales sobre el espiritu empresarial?

\section{Metódología}

El estudio se fundamentó en un enfoque complementario (Bericat, 1998; Montero 2006), con dos momentos de la investigación, para la búsqueda del sentido de las representaciones sociales sobre el espíritu empresarial. En este estudio, la indagación y la comprobación de las evidencias se establecen a partir de los interrogantes e interpretaciones del investigador sobre el quehacer actual en los actores, a través de las técnicas de las entrevistas semiestructuradas y el análisis de contenido. (ver Tabla 1: ficha técnica de la investigación).

\section{Actores}

Prensa: 65 artículos abordaron el espíritu empresarial e Iniciativa empresarial en los periódicos El Nacional y El Universal. Período: Año 2006 al primer semestre del año 2009.

Informantes clave: 10 - 5 Gerentes educativos especialistas en representaciones sociales y espíritu empresarial. 5 Coordinadores de medios y jefes de páginas de periódicos.

Métodos de análisis: Análisis de contenidos del corpus de artículos. Entrevistas a informantes clave.

Tabla 1: ficha técnica de la investigación

El primer momento de la investigación estuvo conformado por la determinación y análisis del corpus el cual quedó constituido por 666 artículos, producto de la búsqueda inicial de las palabras clave. Se realizó sobre la difusión del evento espíritu empresarial y su caracterización en los medios impresos venezolanos: El Universal, periódico fundado en el año 1909 y El Nacional en el año 1943, ambos diarios de circulación nacional. Inicialmente se determinaron todas las entradas que dieron las palabras claves: emprendedor y espíritu empresarial, lo que proporcionó un primer dato de 666 artículos, que fueron segmentados como noticias, artículos de opinión. Y luego fueron contrastados con los documentos físicos que poseía el investigador. En una segunda fase se seleccionaron, a través de lectura, los artículos que tanto en el título como en su contenido y su discusión temática presentaron eventos relacionados sobre la temática estudiada lo que definió el total de 65 artículos que se tomaron como corpus para este estudio.

El período estudiado abarcó los años 2006 - 2009, dado que es a partir del 2006 que los archivos de los dos periódicos están digitalizados lo que facilitó 
la comparación de los registros físicos de cada periódico con la información digital. Los pasos que se siguieron en la investigación fueron: 1) identificación del corpus, 2) construcción de significados de espíritu empresarial con base a la conceptualización de Moscovici, 3) establecimiento de jerarquías con base a la regularidad, estabilidad de los conceptos, pertinencia y las frecuencias de cada dato, 4) el análisis de contenidos de la data recolectada permitió determinar las categorías centrales y periféricas.

Las categorías fueron establecidas por el investigador a partir de diferentes vías, entre las que se mencionan: la revisión teórica y conceptual del objeto de estudio, otros sistemas categoriales previos formulados por otros autores y el éxito previo obtenido con su construcción, opiniones de expertos y especialistas en el objeto de estudio.

Se seleccionaron las categorías con énfasis en el contexto y se siguieron los pasos señalados por Krippendorff (1990), formulación de los datos, determinación de las unidades, muestreo, registro reducción de los datos, inferencias y análisis para lo cual se recurrió a validación directa, verificación de la información. Las categorías más representativas se constituyeron por tópico de la investigación. Para el registro se siguieron los sugerencias dadas por Pińuel (2002) para manejar grandes conjuntos de material de manera estadística, parece indispensable reducir las alternativas que deben disponerse en tablas y esto puede lograrse situando en una categoría una amplia variedad de estructuras de palabras diferentes. El análisis frecuencia permitió observar los aspectos o eventos comunes y divergentes presentes en el corpus analizado.

Se revisaron los textos de los artículos que comprendían las palabras clave: emprendedor y espíritu empresarial. Los textos fueron clasificados por año y se hizo una segunda lectura para determinar categorías abiertas y centrales. Las categorías iníciales fueron obtenidas a través de la identificación de las palabras claves, que permitieron la construcción de núcleos de significados. Estas fueron: espíritu empresarial; Emprendedor social, Emprendedor en: Venezuela, Pymes, educación, tecnología; Emprendedor e informalidad; Emprendedor y rasgos; Emprendedor y creatividad.

El segundo momento de la investigación se focalizó en las entrevistas a los actores que participaron en el estudio: cinco (5) gerentes educativos en el área de espíritu empresarial con publicaciones sobre el tema; tres (3) coordinadores de medios y dos (2) editorialistas que escriben sobre el tópico. Los actores ocuparon un lugar determinante en la pesquisa, sólo a través de ellos se logró comprender las representaciones sociales del espíritu empresarial. 
El proceso de categorización se inició con la recolección de los datos, especialmente la transformación de los datos descriptivos en datos interpretativos y analíticos. La categorías, fueron generadas a través del análisis del corpus y de las verbalizaciones obtenidas en las entrevistas. En el análisis se obtuvieron categorías por "afirmación" que describen los atributos socialmente valorados; y por "oposición”, que describen diferentes tipos de conducta.

El proceso de categorización estuvo relacionado con la forma como se clarifico, conceptualizo o codificó un término o expresión en forma clara o inequívoca (categoría descriptiva), y el contenido o idea central de cada unidad temática. Las categorías establecidas fueron seleccionadas, de acuerdo a la opinión de la mitad de los entrevistados como "nucleares", y las "periféricas" aquellas que no fueron tan frecuentemente referidas por los informantes. En este sentido, se hizo necesario definir y explicar el significado que los protagonistas le otorgaron a las representaciones sociales del espíritu empresarial desde su propia perspectiva. Posteriormente, se procedió a organizar la información, de acuerdo con las categorías identificadas a fin de iniciar el análisis articulado de la información, relacionando los elementos de la categorización con las tendencias identificadas para lograr comprensión integral de las significaciones imaginarias detectadas, permitiendo mostrar un cuerpo de representaciones sociales sobre el espíritu empresarial.

\section{Presentación y análisis de resultados}

Las unidades de análisis fueron los titulares y se registró la lexicalización, entendida como cambio o perdida representativa del significado de una palabra. En nuestro caso los artículos analizados presentan el uso del término de emprendedores un 92,3\% mientras que la denominación espíritu empresarial es usada solo el 7,7\%, a pesar ser la terminología que recomienda la Real Academia Española de la lengua (ver Tabla 2: categorías derivadas del análisis de contenidos del corpus prensa). El término "emprendedor" está usado con las siguientes acciones verbales "emprender", "aprender a emprender", "para emprender"; otros usos son a través de la adjetivación: "jóvenes emprendedores", "emprendedores sociales", "pequeńos y medianos emprendedores", "enseñanza emprendedora”, "mujeres emprendedoras" (denominaciones tomadas de los titulares de prensa).

En cuanto a la acción que realizan los "emprendedores" en Venezuela es conceptualizada a través de las diez palabras más usadas en la prensa: "es una forma de alcanzar metas", "el venezolano tiene ideas", "lleva a cabo ideas o sueños", "tiene capacidad de crear". O como expresa el titular: "Hacer realidad lo soñado", cuando señala que el emprendedor "reúne todos los esfuerzos para materializarla". 
El espíritu empresarial desde las representaciones sociales: caso Venezuela

\begin{tabular}{|c|c|c|c|c|c|c|}
\hline \multicolumn{2}{|c|}{ Categorías } & 2006 & 2007 & 2008 & 2009 & Total \\
\hline \multicolumn{2}{|c|}{ Espiritu empresarial } & - & 1 & 2 & 2 & 5 \\
\hline \multirow{2}{*}{$\begin{array}{l}\simeq \\
0\end{array}$} & Social & 9 & 11 & 4 & 10 & 34 \\
\hline & En Venezuela & - & 4 & 6 & 1 & 11 \\
\hline \multirow{2}{*}{$\begin{array}{l}a \\
\text { II } \\
0\end{array}$} & En Pymes & - & - & 1 & 1 & 2 \\
\hline & En Educación & - & 3 & 1 & - & 4 \\
\hline \multirow{2}{*}{$\begin{array}{l}Z \\
\text { ㅍ } \\
\simeq\end{array}$} & En Tecnología & - & - & - & 2 & 2 \\
\hline & En informalidad & - & - & 1 & - & 1 \\
\hline \multirow{2}{*}{$\bar{\Sigma}$} & Y rasgos & - & - & 1 & - & 1 \\
\hline & Y creatividad & - & 1 & 2 & 2 & 5 \\
\hline \multicolumn{2}{|r|}{ Totales } & 9 & 20 & 18 & 18 & 65 \\
\hline
\end{tabular}

Tabla 2: categorías derivadas del análisis de contenidos del corpus prensa

Vidal (2007) expresa que, según cifras del Monitor Global Entrepreneurship (GEM, en inglés), un estudio realizado en Venezuela por el Centro de Emprendedores del IESA:

[...] casi el $48 \%$ de los venezolanos adultos sońamos con ser empresarios. Además, muchos son los valientes que emprenden la marcha: $25 \%$, de ellos están emprendiendo acciones para montar su compañía o tiene una recién nacida. Esto nos coloca en el primer lugar de iniciativa empresarial de una muestra de 35 países .Ese ímpetu emprendedor viene acompañado de una gran confianza en las habilidades y capacidades personales, para llevar adelante una empresa exitosa. Según el GEM, casi 75\% de los venezolanos considera que cuenta con las destrezas, los conocimientos y la experiencia que se necesitan para iniciar un nuevo negocio.

La primera representación social, está referida a las características del espíritu empresarial del venezolano en aspectos positivos al ser catalogados:

Venezuela ocupa el primer lugar con 25\%, según el índice TEA (Actividad Emprendedora Temprana). Bulgaria ocupa el último lugar con $1,9 \%$. El índice mide la capacidad emprendedora diferenciando entre aquellos emprendedores que lo son porque ven la oportunidad y tratan de aprovecharla, de aquellos que lo son por necesidad. Mientras en Dinamarca existen 27,73 actividades emprendedoras por razones de oportunidad por cada 1 que surge por necesidad, en Venezuela hay 1,65 por oportunidad por cada 1 por necesidad, lo que nos ubica mucho mejor con relación a nuestra realidad. Los resultados del estudio son mucho más importantes, me atrevería 
a decir cuando nos contrastan con nosotros mismos que: $51 \%$ de los emprendedores son mujeres; $47,7 \%$ del total tiene entre 18 años y 34 años de edad; 22,9\% está ubicado en la región Centro Occidental; $20 \%$ en la región Central; $11,3 \%$ en la Nororiental; $11 \%$ en Guayana y $10,6 \%$ en la región Capital. Un último dato que nos retrata de cuerpo entero es que el $63 \%$ de las actividades emprendedoras aspira a un retorno de la inversión en tan sólo un año" (Pizani, 2007).

La Figura 1: representaciones sociales acerca del espíritu empresarial en el venezolano, presenta la forma como la prensa objetiva, los temas del espíritu empresarial. El análisis de los textos hace explicito que la representaciones sociales es nuclear al considerar al venezolano como altamente emprendedor, destacando las referencias a ciertas características en cuanto al género, el nivel educativo, y el sector productivo donde se desarrollan las iniciativas empresariales. En el sentido anteriormente expuesto se observa el anclaje psicológico que está relacionado con las opiniones expresadas por los articulistas y el conocimiento sobre el tema como una forma de interpretar y comprender la realidad. Entre las iniciativas empresariales señaladas en la prensa se reflejan la tendencia al desarrollo de actividades en las siguientes áreas:

Con alimentos $(30,3 \%)$ bodegas y ventas de víveres, repostería, chucherías; restaurantes, comida rápida, panadería, heladería), y los siguientes tres grupos son comercios, ventas de ropa y artículos del hogar (9,5\%); telecomunicaciones, Internet, cibercafés, comunicaciones $(7,3 \%)$ y peluquería, estética, masajes $(3,8 \%)$; acumulando entre todos más del $50 \%$ de los nuevos negocios del sector (Fernández, 2008a).

Los medios juegan un papel decisivo en la difusión y transformación de la cultura empresarial y las practicas del emprendedor, en los cuales encontramos unos procesos de anclaje al considerar a los venezolanos como altamente emprendedores. Sin embargo, esa representacion social está disociada de la conceptualización presente en la literatura especializada sobre el tópico de inciativas empresariales, caracterizado a través de la realización de actividades altamente productivas y que generan valor en la sociedad. Sin embargo, las características del entorno económico social desdibujan este proceso. 


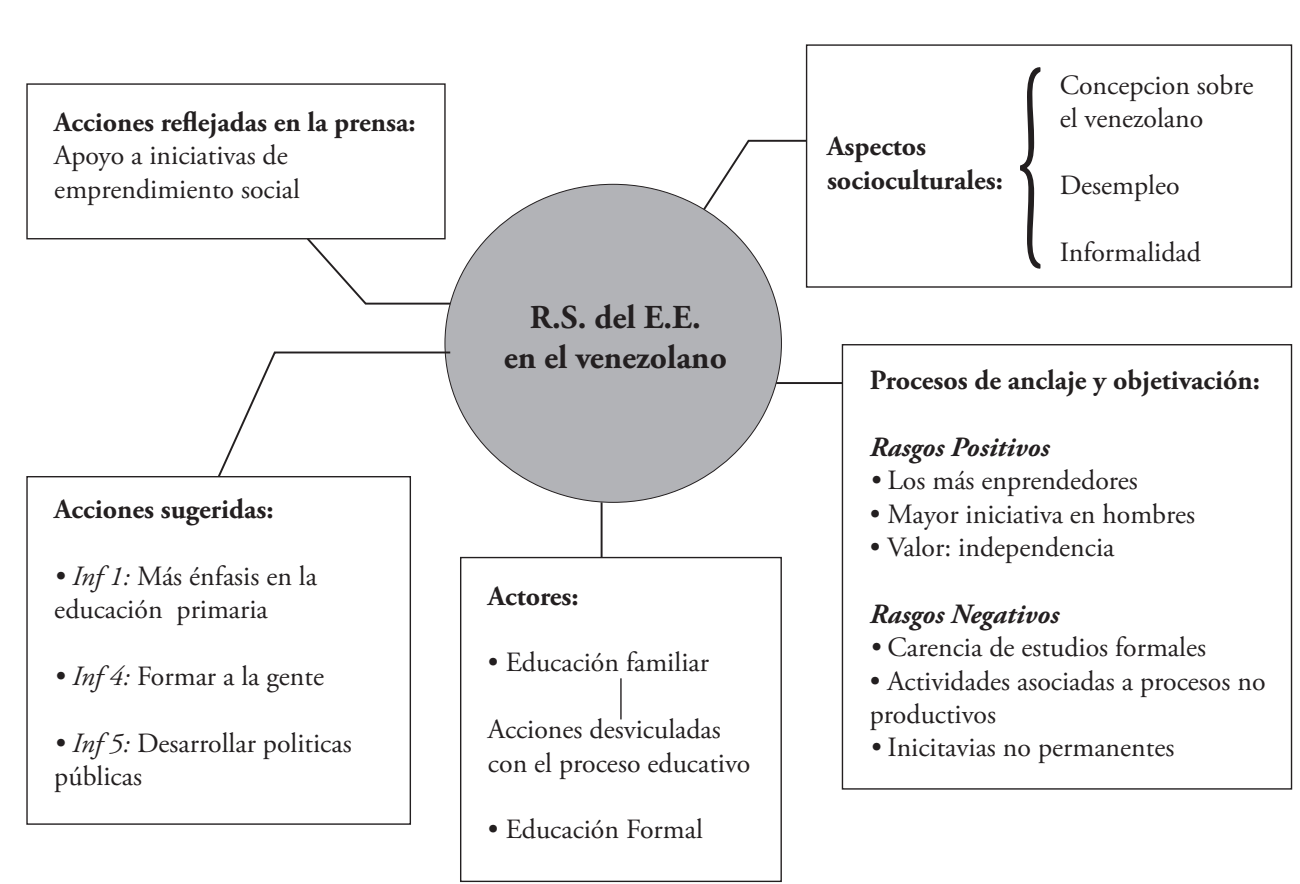

Figura 1: representaciones sociales del espíritu empresarial del venezolano

En cuanto al género, edad y nivel educativo, entre los artículos analizados, Vidal (2008) caracteriza a los a los integrantes del género masculino en función del género como con mayor emprendimientos:

Por primera vez, el estudio muestra en Venezuela mayor iniciativa empresarial en la población masculina que en la femenina, mientras la mayor concentración de emprendedores se encuentra entre los 35 y 44 años de edad. Además, los índices más elevados se encuentran en los extremos de nivel educativo y estrato socioeconómico: los más emprendedores son quienes no completaron la educación básica o quienes tienen estudios de posgrado, así como los que presentan más elevado o más limitado poder adquisitivo (Vidal, 2008).

El análisis de las representaciones sociales develadas por los informantes 1, 2 y 3 sobre la representaciones sociales de su acción, definen al venezolano con intenciones positivas hacia el espíritu empresarial.

Informante 1: "manifiesta que al venezolano le cuesta dejarse abatir por las circunstancias de crisis, trata de hacer algo. Vender café, esto no es lo más conveniente pero saca provecho".

Informante 2: "sostiene que con los pocos recursos se las ingenia". 
Informante 3: "Considero más emprendedora a la mujer, siempre está ingeniándose como llevar el sustento a la familia, será por eso que las principales iniciativas, están asociados con actividades predomínate del género femenino".

Los informantes 4, 5, 6 y 7, caracterizan la presencia del espíritu empresarial de la siguiente forma:

Informante 4: "se observa que existe un porcentaje muy pequeño de venezolanos con competencias personales. Sin embargo, el porcentaje más alto parece tener impulsos esporádicos para iniciar empresas o negocios privados o mixtos."

Informante 5: "el venezolano tiene una tendencia al facilismo a la práctica del mínimo esfuerzo, no corre riesgos en cuanto al desarrollo de empresa, para otros eventos artísticos si lo tienen. Hay una comodidad, por lo que hemos vivido por la dependencia del Estado, fíjate, el venezolano no es flojo, porque se levanta a las 4 am. Es cómodo, el petróleo nos hizo así. Para ir más lejos, para qué nuestros indígenas iban a esforzarse, si desde el chinchorro podían tirar el anzuelo y pescaban".

Informante 6: "quiero pensar que tenemos el talento humano, sin embargo las pocas oportunidades que se dan nos llevan a que nos califiquen como los más emprendedores, sin embargo no lo veo en la práctica quiero decir con el nivel de desempleo y marginalidad".

Informante 7: "el venezolano es emprendedor, siempre es ingenioso, pero no es planificado, ni consistente, por lo que no sostiene sus iniciativas".

Si consideramos que el mundo se construye por las prácticas socioculturales y el comportamiento de los individuos, y que las representaciones sociales son modalidades de la vida de los individuos y circulan en las conversaciones, en los discursos mediáticos, se cristalizan en las conductas y en las acciones, pudiéramos develar dos tipos de representaciones sociales: una expresada en los medios acerca del venezolano como emprendedor producto de estudios derivados del Global Entrepreneurship Monitor (GEM) realizado por el IESA; y otra que caracteriza al venezolano "como uno de los más emprendedores del mundo".

Otra representación social nuclear es el emprendimiento social que aborda diferentes tópicos en actividades para apoyar el tejido empresarial por parte de organismos públicos y privados. En esta representación social prevalece un nuevo valor que es la convivencia social en el medio productivo. Es una posible solución 
a los problemas y conflictos presentes en el ambiente venezolano. Sin embargo esta acción no se refleja con resultados a nivel económico, en indicadores como creación de empleo y crecimiento tecnológico, bienestar para la sociedad por el aporte a la economía, al generar riqueza y empleo (ver Tabla 1 ).

En cuanto a la caracterización del evento espíritu empresarial en la prensa nacional, el cómo los informantes perciben la construcción de la representaciones sociales sobre espíritu empresarial, llama la atención la coincidencia de opiniones de los informantes 1, 2, 4, 5 y 7 acerca de la poca difusión en los medios estudiados:

Informante 1: "Yo como lector empedernido, considero que este tópico es mínimo en cuanto aniveles de profundidad y alcance en los medios de comunicación impresos nacionales".

Informante 2: "... es poca información sobre este tópico en la prensa, consigo más información en revistas especializadas e Internet”.

Informante 4: "Se están dando los primeros pasos. No está aun consolidado, hace falta mayores logros entre los ciudadanos, los medios de comunicación y con un mejor contenido. Estas son nuestras aspiraciones".

Informante 5: "la información es escasa porque actualmente apenas recién se inicia el concepto sobre espíritu empresarial. No es claro, en algunos trabajos veo que lo mezclan con responsabilidad social".

Informante 7: "leo diariamente la prensa en búsqueda de elementos que pudieran darme información. Veo poca cobertura y lo que hay es poco claro. También me llama la atención que el término es usado como un comodín en avisos de reclutamiento, personal, obituarios".

\section{Conclusiones}

Las conclusiones derivadas del estudio se insertan en la comprensión del evento representaciones sociales sobre el espíritu empresarial, que están presentes en los informantes clave y las derivadas del estudio de los medios impresos, en las cuales se objetiva al venezolano como uno de los más emprendedores. El proceso de objetivación examinado a través de la representación social sobre el emprendimiento del venezolano explica cómo está imbricada la creencia del venezolano como sujeto emprendedor, lo cual permite afirmar que existe un anclaje acerca de las conductas emprendedoras debido a efectos contextuales presentes en el medio venezolano como son la crisis, el nivel desempleo, que llevan a caracterizar al venezolano como emprendedor por necesidad en vez de emprendedor por oportunidad. Estos hallazgos son coincidentes con los develados 
por Chirinos y Guerrero (2009: 72 y 73) en cuanto a la presencia de espíritu empresarial en el venezolano.

La tendencia de los articulistas y editorialistas acerca de este hecho pareciera incentivar estas experiencias al impulsar el espíritu empresarial en el colectivo social. Se recomienda estudiar en futuras investigaciones el espíritu empresarial en el desarrollo de las comunidades con iniciativas diferentes a las reseñadas en el cuerpo de este trabajo, como una forma de transformar las organizaciones públicas o privadas a fin de entender, según Jodelet (2004), cómo el pensamiento social construye la realidad actual en nuestras sociedades, la información se liga al quien más sabe.

El estudio de representaciones sociales, agrega una información en cuanto al anclaje mediante el cual lo no "familiar "o remoto es asimilado en las categorías de la cognición cotidiana, que guía la conducta colectiva al pasar un constructo del dominio científico al dominio público como una forma de interpretar la realidad y la objetivación que hace que las representaciones se proyecten en el mundo. Así, lo que está a nivel de ideas se transforma en concreto en los discursos de la prensa nacional y en lo "que sé" y lo "que creo" es evidenciado por los informantes claves en sus representaciones sociales acerca del espíritu empresarial. Esta aseveración se realiza por la forma como es usado el término espíritu empresarial en la prensa.

En opinión del autor es necesario resaltar acciones en los diferentes actores que intervienen en el proceso de educación empresarial, en cuanto al desarrollo del espíritu empresarial, que de acuerdo a estudios realizados por el Global Entrepreneurship Monitor (GEM) en Venezuela, señala: altas tasas de espiritu empresarial en los venezolanos, que no son coherentes con los resultados de crecimiento del país a fin de establecer mecanismos que permitan formar en el discurso de los actores significados compartidos, porque las representaciones sociales, son conceptos, nociones, actitudes, que están imbricadas en el lenguaje para explicar a nivel teórico las construcciones del pensamiento individual en un discurso colectivo. Se necesita mayor difusión acerca de la conceptualización, importancia, necesidad del estudio como competencia del espíritu empresarial, desde la educación inicial como una de las vías de mejora en las condiciones socio económicas del venezolano, así como la transformación de las representaciones sociales del espíritu empresarial en el medio venezolano.

La fase sintética derivada de la aplicación de la complementariedad buscó hacer de manera explícita el uso del análisis de contenido que identificó en los artículos analizados, unidades claves que aparecieron en forma consistente $\mathrm{u}$ otras que son sólo situaciones periféricas de la representaciones sociales sobre el espíritu empresarial. 
La prensa nacional influye en los intereses y motivaciones de los lectores, en este caso la presencia de un liderazgo en lo social que conlleva la caracterización de experiencias, vivencias, que han sido realizadas con el apoyo de iniciativas privadas y en menor cuantía del gobierno local. La representaciones sociales, está caracterizada por la prevalencia cuantitativa en el caso de la acción reflejada en los medios, con alta tendencia (53\%) hacia el emprendimiento social. Otro núcleo de representaciones sociales, que fue determinado consensualmente es la caracterización de los informantes sobre la presencia de espíritu empresarial en el venezolano, el cual lleva a repensar una consideración acerca de qué se está entendiendo por espíritu empresarial.

En otro orden de ideas hay una representaciones sociales sobre la poca durabilidad de la iniciativas desarrolladas, situación agravada por la carencia de políticas formales que apoyen el desarrollo del proceso del espíritu empresarial en todos los niveles organizativos y empresariales ante una realidad que presenta la implantación de un modelo político que aún no termina de aceptarse en el aparato productivo venezolano.

El análisis evidenció la necesidad de profundizar la información acerca del desarrollo del espíritu empresarial en el contexto, a través de acciones compartidas entre los diversos actores que intervienen en el acontecer venezolano: gobierno, inversionistas, formadores en educación empresarial, empleadores y medios de comunicación, para constituir una cultura que favorezca mejores oportunidades de vida y desarrollo socio económico.

En futuras investigaciones es necesario estudiar la presencia de la cultura nacional (Borjas y Monasterios, 2009), la cual está caracterizada por patrones desalentadores hacia el trabajo.

\section{Bibliografía}

Abric, C. (2001). Metodología de recolección de representaciones sociales. En Practiques socials et representations. Traducción al español por José Dacosta y Fátima Flores. Edic. Coyoacán. México

Banchs, M. (2005). Aproximaciones procesuales y estructurales del estudio de representaciones sociales. Disponible en: www.psr.jku.at/PSR2000/9_3Banch.pdf.

Bericat, E. (1998). La integración de los métodos cuantitativo y cualitativo en la investigación social: Significado y medida. Editorial Ariel, Barcelona. 
Borjas L. y D. Monasterios (2009). representaciones sociales de la ética empresarial en el contexto latinoamericano (Caso: Venezuela), Cali, Colombia. Revista CES, Facultad de Derecho, ICESI, Santiago de Cali, Colombia, \# 4.

Borjas L., Eugui, M, Marvez, G y .Monasterios (2010). Una análisis de contenido de la Iniciativa empresarial en una institución de educación superior venezolana, Revista Anales de la Universidad Metropolitana, Caracas, trabajo en prensa

Chirinos, J. y A. Guerrero (2009). representaciones sociales de los docentes y empleadores sobre la enseñanza y competencias del espiritu empresarial presentes en los egresados de dos universidades venezolanas, Caracas, Venezuela, Universidad Metropolitana, Tesis de grado para optar al título de Lic. en Ciencias Administrativas. Mención Publicación.

Fernández, F. (2008a).Venezolanos emprendedores. El Universal, Caracas.

Fernández, F. (2008b). Hacer realidad lo soñado. El Universal, 30/127 2008, Caracas.

Hayton, J y Kelley. D. (2006). A competency-based framework for promoting corporate entrepreneurship. Human Resource Management, VL: 5, No: 3, pp. 407427. Donna Bocconi University, Milan.

Jodelet, D. (2001). As representacoes sociais. Rio de Janeiro, Editora de Universidad DoRio de Janeiro.

Jodelet, D. (2004). Vigencia de las representaciones sociales y su incidencia en las prácticas profesionales. Educar, Argentina. Disponible en portal.educ.ar/noticias/ entrevistas/dra-denise-jodelet-vigencia-de.php-58k-Consultado l mayo 2007.

Kantiss, H, Angelelli, M y Moori, D.( 2004).Desarrollo emprendedor en América Latina, Buenos, Aires, BID.

Krippendorff, K. (1990). Metodología de análisis de contenido. Teoría y Práctica. Buenos Aires. Paidos Comunicación.

Montero, M. ( 2006). Hacer para transformar. El método en la Psicología comunitaria. Buenos Aires, Editorial Paidós.. 
Moscovici, S. (1973). Prólogo a C. Herzlich, Health and Illnesss. Londres, Aca-

demic Press.

Moscovici, S. (1984). The phenomenon of social representations, en R.M. Farr y S. Moscovici (comp.), social Representations, Cambridge, Cambridge University Press, pp. $3-69$.

Pizani, L. (2007). Los más emprendedores del mundo, Caracas, El Nacional. Caracas. http://www.guia.com.ve/noticias/?id=2335

Piñuel J.L (2002). Epistemología, metodología y análisis de contenido, Estudios de Sociologia, No 3,1. Pp. 142.

Vasilachis de Gialdino. I. (2003). Pobres, pobreza, identidad y representaciones sociales, Barcelona, Gedisa Editorial.

Vidal, R. (2007). A la caza del mejor negocio del mundo. El Universal, Caracas, 17-04-2007, cuerpo 1-8.Disponible en: http://www.eluniversal. com/2007/04/17/opi 42919 art a-la-caza-del-mejor 239012.shtml

Vidal, R. (2008a). Emprender no basta. El Universal, Caracas, 26-02-2008, cuerpo 1-8.

Vidal, R. (2008b). Quién emprende en Venezuela. El Universal, Caracas, 0804-2008. Disponible en: http://www.eluniversal.com/2008/11/11/proem blog quien-emprende-en-v $11 \mathrm{~A} 2123405 . \mathrm{shtml}$ 
